\title{
Ambulatory dispersal in Tetranychus urticae: an artificial selection experiment on propensity to disperse yields no response
}

\author{
N. S. H. Tien • M. W. Sabelis $\cdot$ M. Egas
}

Received: 22 June 2010/ Accepted: 24 October 2010/Published online: 9 November 2010

(C) The Author(s) 2010. This article is published with open access at Springerlink.com

\begin{abstract}
Dispersal to new hosts is an important process for an invasive herbivore, such as the two-spotted spider mite. A recent study, using artificial selection experiments, has suggested that genetic variation and genetic trade-offs are present for propensity to disperse in this species. However, due to the experimental setup alternative explanations for the response to selection could not be ruled out. Using an altered setup, we investigated whether the propensity for ambulatory dispersal differs genetically between individuals and whether genetic correlations with life-history traits exist. Upward and downward selection on propensity to leave the colony was performed for seven generations in four replicate artificial selection experiments and the results were compared to control lines. No consistent responses to selection were found and no significant effect on life-history traits (oviposition rate, juvenile survival, development rate and number of adult offspring) or sex ratio was present across the replicates. The data suggest that our base population of spider mites harbours at best a low amount of additive genetic variation for this behaviour.
\end{abstract}

Keywords Two-spotted spider mite $\cdot$ Artificial selection · Genetic trade-offs · Dispersal $\cdot$ Life-history $\cdot$ Colonization

Electronic supplementary material The online version of this article (doi:10.1007/s10493-010-9411-7) contains supplementary material, which is available to authorized users.

N. S. H. Tien - M. W. Sabelis · M. Egas (ه)

Institute For Biodiversity and Ecosystem Dynamics, University of Amsterdam, P.O.Box 94248,

1090GE Amsterdam, The Netherlands

e-mail: C.J.M.Egas@uva.nl

N. S. H. Tien

e-mail: N.S.H.Tien@uva.nl

M. W. Sabelis

e-mail: M.W.Sabelis@uva.nl 


\section{Introduction}

Many mite species live in transient habitats (Mitchell 1970; Kennedy and Smitley 1985). The herbivorous two-spotted spider mite, Tetranychus urticae Koch, is subject to high spatial and temporal variation in host plant availability and suitability. It can quickly overexploit a host due to its high intrinsic rate of increase (Sabelis 1991). Also, predators such as Phytoseiulus persimilis can eradicate local colonies of spider mites and survival of the spider mite is then dependent on dispersal to neighbouring plants (Ellner et al. 2001). Tetranychus urticae is recorded to feed on hundreds of plant species (Jeppson et al. 1975; Bolland et al. 1998), but its performance differs greatly between hosts and unfavourable hosts are often abandoned (Fry 1989; Yano et al. 1998). The mite also occurs globally as a pest in agriculture and horticulture (Jeppson et al. 1975; Helle and Sabelis 1985), where frequent crop harvesting and pesticide treatment are part of its environment and its dispersal rate varies greatly throughout the season (Hussey and Parr 1963; Brandenburg and Kennedy 1982; Margolies and Kennedy 1985). Hence, dispersal is an essential process in the ephemeral habitats colonized by $T$. urticae.

Metapopulation theory suggests that in species that live in unstable environments, dispersal traits may be strongly related to life-history evolution, especially reproductive traits (e.g., Olivieri et al. 1990; Ronce and Olivieri 1997). Empirical studies have demonstrated the presence of genetic correlations between dispersal and reproductive traits in various species (e.g., Roff and Fairbairn 2001; Fjerdingstad et al. 2007; Roff 2002). For the case of $T$. urticae, recent work suggested a genetic trade-off between dispersal and reproductive traits in this species (Yano and Takafuji 2002). Yano and Takafuji (2002) performed artificial selection on the propensity for ambulatory dispersal, using a Japanese population of T. urticae. They found differences in dispersal behaviour between upward and downward selected lines. Also, the upward selected lines exhibited lower rates of reproduction on some host plant species (albeit not on their original host), which suggests a genetic trade-off may be present between dispersal and reproduction-related traits. However, the experimental set-up used may have led to other factors differentially influencing the genetics of the selection lines. The two selection regimes differed in more than one aspect from each other. Upward selection involved the choice between staying on an overexploited dying leaf and walking over a bridge to a new food patch, and the first 20 females that dispersed via the bridge were chosen to produce the next generation. Downward selection involved the choice between staying on an overexploited dying leaf and walking into wet cotton wool (leading to a certain death), where the last 20 females that remained on the leaf were chosen to produce the next generation. This latter treatment not only selects against the propensity to walk into the water barrier, but also selects for immobile adult females, which likely have different genetic bases and non-genetic bases (e.g. disease) from propensity to leave the colony. Also, population sizes of the lines were very low (20 females) and will have led to inbreeding and possibly drift (despite an absence of significant differences between replicate lines in their statistical models). A combination of these factors may account for (part of) the reported differences in direct and indirect responses in the two treatments in their experiment. Since no control lines were available, it is also not possible to determine which of the two treatments actually resulted in a change.

Here, we report on a new set of experiments using a modified experimental set-up that takes these problems into account. We followed Yano and Takafuji's protocol, but changed three central elements: (1) the population size was made larger to minimize potential inbreeding (2) downward selection was exerted on dispersing individuals instead of 
non-dispersing individuals, and (3) control lines were maintained in order to control for inbreeding effects, environmental fluctuations and to differentiate between the responses to the two directions of selection. Also, we examined a wider suite of life-history traits in the resulting selection lines.

\section{Materials and methods}

In September 2008 a sample of more than 500 spider mites was taken from a population along a transect of $5 \mathrm{~m}$ of spindle bushes (Euonymus europaeus) in the dunes along the Dutch coast near the town of Castricum. This population was maintained (minimal population size $=500)$ in the laboratory under climate-controlled conditions $\left(17^{\circ} \mathrm{C}, 60 \%\right.$ humidity, $\mathrm{L}: \mathrm{D}=16: 8$ ) for 3.5 months before the start of the experiment. The spider mites were cultured on bean leaves (Phaseolus vulgaris), which were placed on wet cotton wool surrounded by water in open plastic containers. They were provided with a surplus of food and space, in order to minimise the tendency to disperse. This apparently worked, since we found no individuals that had walked into the wet cotton wool surrounding the leaf discs. No wind source was present, which suppresses the initiation of aerial dispersal behaviour (Smitley and Kennedy 1985). The climate-controlled conditions during the experiments were $55 \%$ humidity, $\mathrm{L}: \mathrm{D}=16: 8$, and $26^{\circ} \mathrm{C}$.

\section{Selection}

We performed artificial selection on the propensity to leave a colony. The colony consisted of an age-cohort of mites that inhabited a detached bean leaf, placed on wet cotton wool surrounded by water in an open plastic container. As the mites developed, the leaf became increasingly exploited and thus increasingly unfavorable as a food source. This leads to increasingly favorable environmental conditions for dispersal. To disperse, the mites had to cross a hostile environment (a plastic bridge over wet cotton wool) before they reached a suitable habitat (a fresh bean leaf). Crossing the bridge is interpreted as an act of dispersal away from the exploited leaf, i.e. leaving the colony, rather than an act of dispersal towards a newly detected habitat, because in this type of set-up the new habitat does not attract $T$. urticae females (Yano et al. 1998). Hence, the fresh bean leaf served to arrest mites dispersing away from the exploited habitat.

Since dispersing spider mites mainly consist of pre-ovipositing adult females (Brandenburg and Kennedy 1982), we selected on these females. The experiment consisted of three treatments; (a) selection on females with the highest dispersal rate- the high lines(b) selection on females with a low dispersal rate - the low lines-and (c) no selectionthe control lines. Each treatment was replicated four times, resulting in twelve experimental populations which were maintained simultaneously. All populations were initiated by placing 65 mated females on a detached bean leaf. The bean leaves used were first leaves of circa two-week old bean plants and were of roughly similar size and shape. After $24 \mathrm{~h}$ the females were removed and their eggs left to develop. The chosen number of females and the size of the leaf were found in pilot experiments to result in a highly exploited state of the leaf around the time their offspring reached adulthood (personal observation $\mathrm{N}$. Tien). In agreement with this observed timing of overexploitation, the development time of the offspring was found to be similar to that of offspring offered ample food supply (unpubl data N. Tien). Hence, the leaf did not reach the overexploited state for the time the individuals spent in the juvenile phase. 
Before the offspring reached adulthood, the leaf was connected to two or three fresh bean leaves by means of plastic bridges per new leaf. A fresh leaf was placed at the tip of the exploited leaf and connected with at least one bridge. Another fresh leaf was placed at the base of the exploited leaf and connected with at least two bridges. If many mites clustered at a certain point on the exploited leaf, an extra bridge to a(n extra) fresh leaf was added to provide these mites with an opportunity to disperse (instead of walking into the wet cotton wool). Each bridge was circa $0.5-1 \mathrm{~cm}$ wide and 3-5 cm long. The leaves were at least $2 \mathrm{~cm}$ distant from each other. Under our experimental conditions, T. urticae cannot move over the wet cotton wool and the females were thus forced to use the bridge for ambulatory dispersal. In the high lines, the earliest-dispersing individuals were selected (at least 65). In the low lines, the first dispersers were culled. The number of culled individuals was decided on per case, depending on the total number of females present; enough females had to remain on the old leaf to allow at least 65 subsequent females to migrate afterwards. In the control lines, at least 65 females were chosen at random from the old leaf. Usually, the number of dispersed females was higher than 65 and of these dispersed females 75-80 were randomly chosen. The females were placed together on a fresh leaf to mature further for a few days (adult females start ovipositing once they are a few days old), after which 65 females were randomly chosen to form the new generation. Seven rounds of selection were performed. Time of day at which the required number of selected females was achieved varied greatly between generations, from early in the morning to late in the afternoon, but no consistent difference was present between treatments or lines (pers. obs. Tien).

After the seventh generation of selection, 75 of the selected females were allowed to lay eggs on a fresh leaf for $24 \mathrm{~h}$. The offspring formed the basis of the resultant selection lines for which dispersal and life-history traits were subsequently determined. These lines were kept on bean leaves placed on wet cotton wool in individual containers and provided with ample food and space. The twelve lines were subdivided into four groups, which were investigated at different points in time to spread the amount of work involved given the limited manpower. Every group contained one line of every treatment. The groups were termed replicates 1-4. Consequently, the populations were named as follows: [name treatment] [number replicate], where the treatment is either high, low or control and the replicate is $1,2,3$ or 4 .

\section{Selection response}

Propensity to disperse was measured with a group-wise dispersal index and an individual dispersal index. For the group index, a cohort of ten adult females of the same age was placed on a fresh leaf disc (diameter $=1.5 \mathrm{~cm}$ ), which was connected to a second leaf disc by a plastic bridge of $5 \mathrm{~cm}$ long. Ten females on a leaf disc of this size cause overexploitation of the leaf disc within a few days, which thus creates a situation roughly comparable to the set-up of the selection experiment. This index is similar to the one used by Yano and Takafuji (2002). Over a period of 3 days, the number of females that were found on the second disc was recorded six times (three times on day one, two times on day two and one time on day three). After every recording, the dispersed females were removed. Females that died were removed from the analysis from that time point onwards. Initial sample size per line was 24, totalling 288 samples (see ultimate sample size per line in Table 1). The data were analysed in two ways. First, the cumulative fraction of dispersers over time was analysed using a mixed-effect generalized linear model with temporal pseudoreplication (see Crawley 2007). In this model treatment was a fixed factor, 


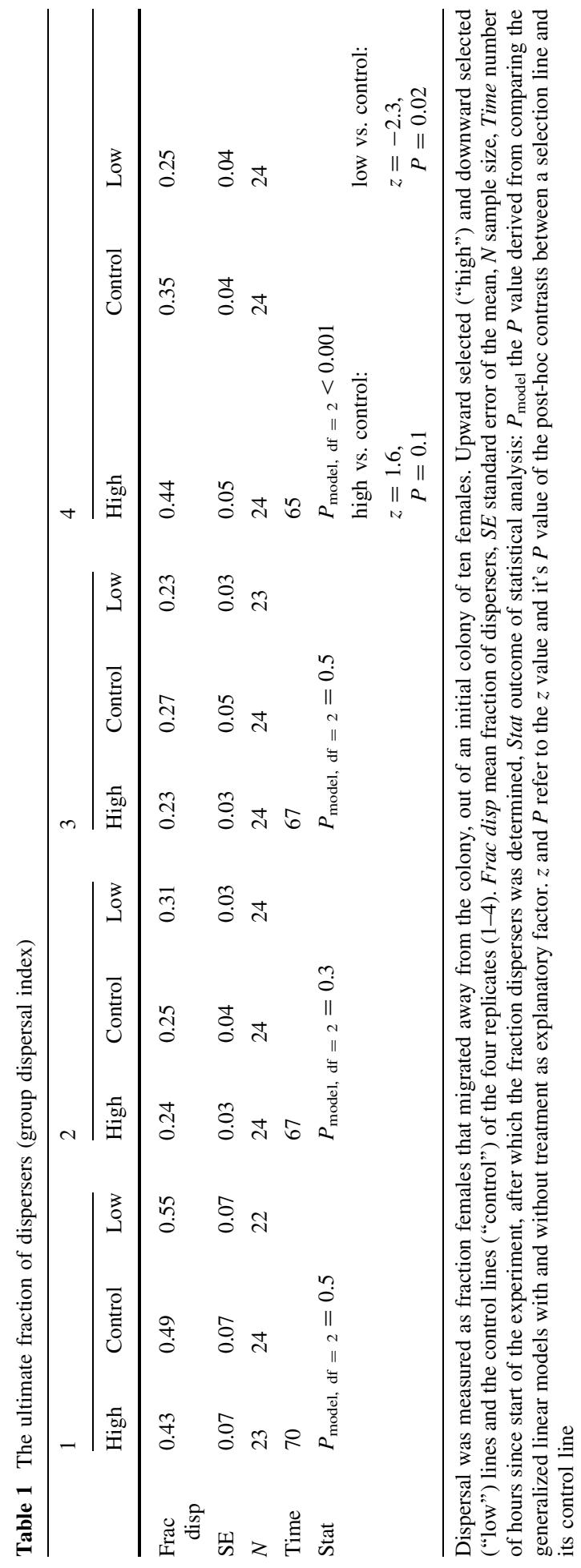


time per sample was a random factor (to correct for the dependency of data points through time within a sample) and a binomial error distribution was assumed (because the data are proportion data). The model with treatment as factor was compared to the model without treatment as factor, using a $\chi^{2}$ test. If treatment was a significant factor, planned comparisons were performed (by using built-in contrasts in the model) between high versus control and low versus control (Crawley 2007). Second, in order to obtain a quantitative measure for dispersal rate, we determined the ultimate fraction dispersers (the fraction dispersers at the last examined point in time). In a generalized linear model the effect of treatment (as fixed factor) on the fraction of dispersers was examined. A binomial error distribution was assumed, except if there was overdispersion (i.e. if the residual scaled variance was more than three times larger than the residual degrees of freedom), in which case a quasibinomial error distribution was assumed in order to scale the variance appropriately (Crawley 2007, Ch. 16, p 327-329). The model with treatment as factor was compared to the model without treatment as factor, using a $\chi^{2}$ test. If treatment was a significant factor, planned comparisons were performed (by using built-in contrasts in the model) between high versus control and low versus control (Crawley 2007). Analyses were performed in R (R-Development-Core-Team 2009) and data were analysed per replicate (as in all analyses).

For the individual index, a set-up was chosen in which leaf deterioration was independent of the presence of the mite. A leaf disc was placed at one end of a rectangular plastic sheet of $5 \times 20 \mathrm{~cm}$, which was placed on wet cotton wool in water. The leaf disc did not touch water and therefore would slowly dry up and wither. At the other end of the plastic rectangle a small bean leaf was placed with its stem in a hole, touching the water, which kept the leaf fresh. An adult female was placed on the desiccating leaf disc. All females were of the same age. The time until the female reached the fresh bean leaf was measured. The first $30 \mathrm{~min}$ the females were monitored continuously, the next $4.5 \mathrm{~h}$ they were checked on the hour. If the mite had not left the leaf disc, they were checked once more after a day. All mites had either migrated or died within this period. Mites that had died on the leaf or walked into the water were discarded from the analysis. The individual index was defined as the fraction of dispersing females that dispersed within $30 \mathrm{~min}$. (Other indices were also examined-e.g. fraction that dispersed within $1.5 \mathrm{~h}$-and similar results were found.) Sample size per line was 18, totalling 216. The data were analyzed with $2 \times 2$ contingency tables and G-tests for independence (Sokal and Rohlf 1995, p 729). The frequencies (dispersed within $30 \mathrm{~min}$ and afterwards) of a selection treatment were compared to those of its control treatment, to see if the fraction early dispersers differed between the two treatments. The $\mathrm{G}$ values were adjusted with William's correction to correct for an inflated type I error (Sokal and Rohlf 1995, p 729).

\section{Correlated responses}

Of the cohorts of offspring created directly after the last round of selection, 25 adult females were randomly chosen per line and placed individually on fresh leaf discs. These females were of the same age (13 days old). The females were allowed to oviposit for $24 \mathrm{~h}$ after which they were removed. The life-history traits of the lines were determined using the offspring. The eggs were counted and on the 9th day the developmental stages of the offspring were determined. Number of female and male adults was counted once all offspring had matured, on the 13th day. Oviposition rate was taken as the number of eggs laid by the female in $24 \mathrm{~h}$. Development rate was represented by an index for the average stage of the offspring on the 9th day after oviposition (where 1 is the egg stage and 7 is the 
adult stage, with five larval and pupal stages in between (cf Tien et al. 2009). Juvenile survival was taken as the fraction of offspring that survived to adulthood. Sex ratio was defined as fraction males in the adult offspring. The data were analysed using generalized linear models in R ( $\mathrm{R}$ Development Core Team 2009). A model with treatment as explanatory factor was compared to the null model with an $\mathrm{F}$ or Chi-square test (Crawley 2007). If treatment was a significant factor, planned comparisons (via built-in contrasts) were performed of control against high and against low. Models that were close to significance $(0.05<P<0.1)$ were also investigated further, to look for trends. Oviposition rate, development rate and number of adult offspring were analysed assuming a normal error distribution. Juvenile survival and sex ratio were analysed assuming a binomial error distribution.

\section{Results}

In the Appendix (Electronic supplementary material) an example of dispersal over time is shown for 9 random lines (without prior selection). This example shows that dispersal predominantly (if not completely) took place during the day and dispersal within a line took place over a period of at least $12 \mathrm{~h}$ and often at least $24 \mathrm{~h}$.

\section{Selection}

In the high lines, out of the total number of adult females the average fraction of selected females per round was 0.28 ( $\pm \mathrm{SE}=0.04)$. In the low lines, an average fraction of 0.44 $( \pm 0.05)$ was first allowed to disperse and then culled, after which an average fraction of 0.26 ( \pm 0.04$)$ was selected (out of the total number of adult females) as the next generation. The average total number of adult females (dispersing and non-dispersing) per round was $373( \pm 32)$ with no consistent difference between the treatments.

Response to selection

When placing ten females on a small leaf disc, within 3 days on average $34 \%$ of the females dispersed to a new leaf disc. The fraction dispersers over time is shown in Fig. 1. The dispersal over time of the high and low lines showed no consistent patterns compared to that of their control line. In replicate $1\left(\chi^{2}=14.6, P<0.001\right)$, the low line had a higher dispersal rate than the control line $(z=2.9, P<0.01)$, while the high line was not significantly different from the control line $(z=-0.8, P=0.4)$. In replicate 2 , there was no significant difference in either treatment $\left(\chi^{2}=1.6, P=0.4\right)$. In replicate $3\left(\chi^{2}=34.8\right.$, $P<0.001)$ both treatments had lower dispersal rates than the control line (high line: $z=$ $-5.0, P<0.001$, low line: $z=-5.0, P<0.001)$. Only in replicate $4\left(\chi^{2}=81.8\right.$, $P<0.001)$ did the lines behave as expected: the high line showed a higher dispersal rate $(z=4.4, P<0.001)$, while the low line exhibited a lower dispersal rate $(z=4.7$, $P<0.001)$. When focussing on the ultimate fraction of dispersers in this experiment, the dispersal rate of the selection lines was not significantly different from that of their control lines in three of the replicates (Table 1). Only in replicate 4 were differences found: The low line had less dispersers than the control line (Table 1). Together, these results show that the differences in dispersal behaviour between the lines were small and not related to selection treatment. 


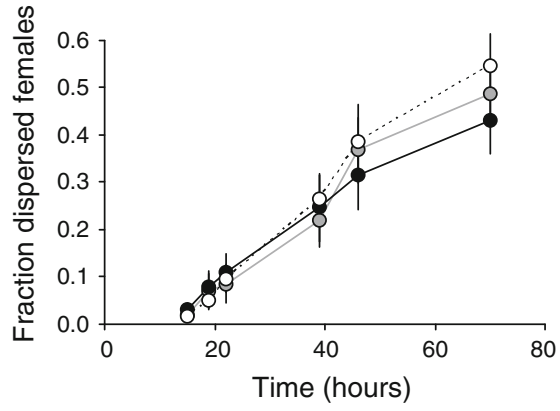

(1)

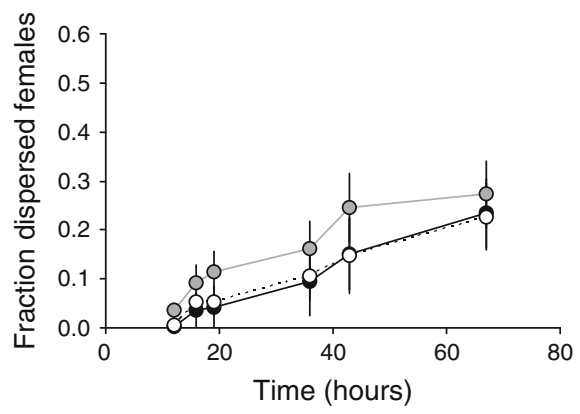

(3)

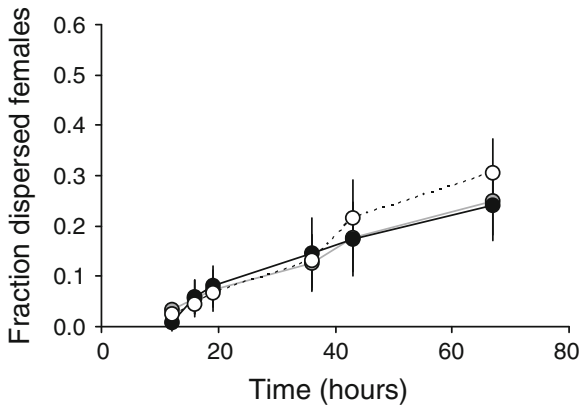

(2)

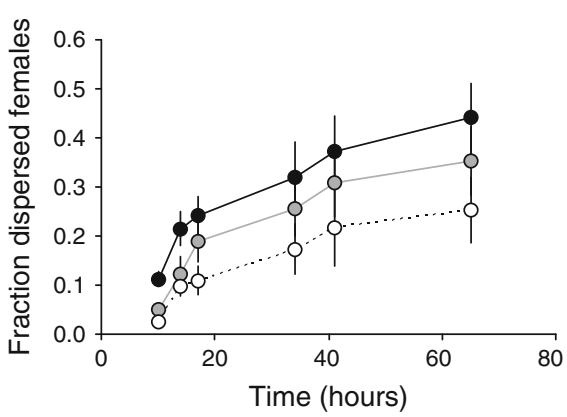

(4)

Fig. 1 Fraction dispersing females over time (group dispersal index). Upward selected (black circles and straight black line) and downward selected (white circles and dashed line) lines and the control lines (grey circles and grey line) of the four replicates (1-4). Dispersal was measured as fraction females that migrated away from the leaf disc, out of an initial colony of ten females. Error bars are standard errors of the mean

When placing a single female on a bad-quality leaf disc, $88 \%$ of the females dispersed to a nearby fresh leaf within a day, while the remaining females died. Figure 2 depicts the fraction of fast dispersers, i.e. the females that dispersed within $30 \mathrm{~min}$ (the individual index). In replicate 1 , the frequency of fast dispersers was not significantly different between the control line and the high line $\left(G_{a d j, 1}=1.70, P=0.19\right)$ or the low line $\left(G_{a d j, 1}=0.01, P=0.92\right)$. In replicate 2 , the high line had significantly less fast dispersers than the control line $\left(G_{a d j, 1}=3.92, P=0.05\right)$, while the low line was not different $\left(G_{a d j, 1}=0.25, P=0.61\right)$. In replicate 3 , the low line had significantly more fast dispersers than the control line $\left(G_{a d j, 1}=4.51, P=0.03\right)$, while the high line was not different $\left(G_{a d j, 1}=0.26, P=0.61\right)$. In replicate 4 , neither selection line was significantly different from their control line (high line: $G_{a d j, 1}=1.74, P=0.19$, low line: $G_{a d j, 1}=0.15$, $P=0.70)$.

\section{Correlated responses}

Neither of the selection treatments led to consistent differences in life-history traits or sex ratio in the selection lines, when compared to their control line (Table 2). Only in two cases were significant differences found; the high line of replicate 2 had a higher sex ratio than its control line and the high line of replicate 3 showed higher juvenile survival. 


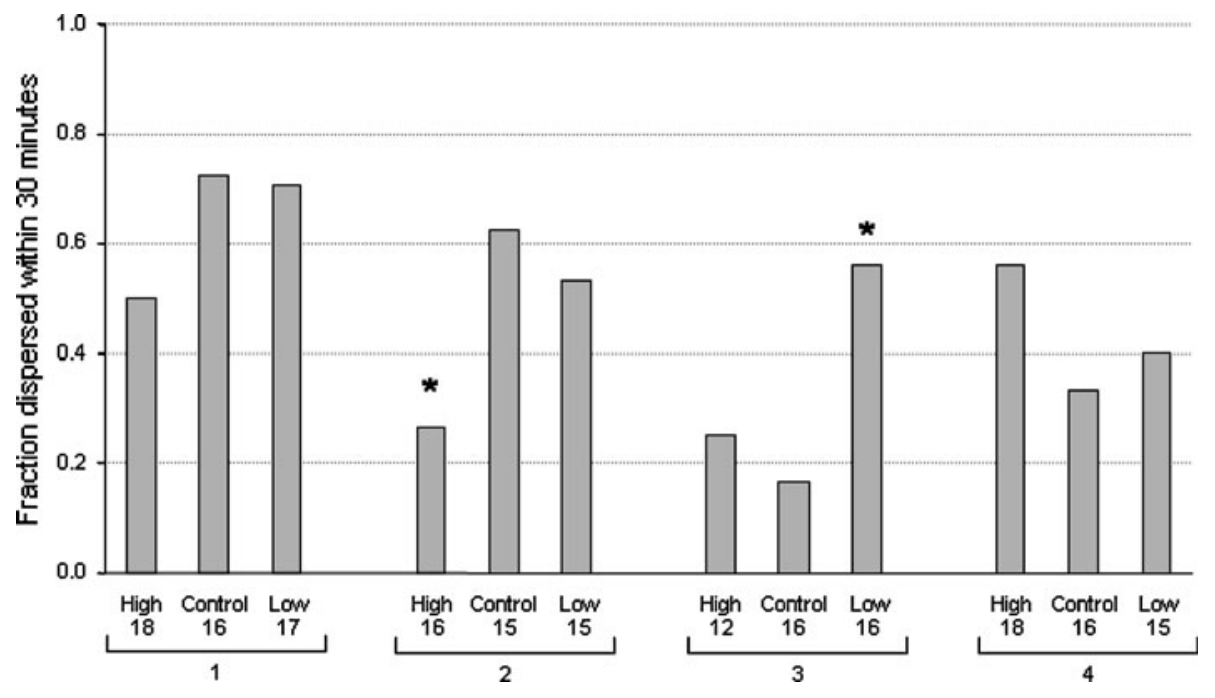

Fig. 2 Fraction of females that dispersed within $30 \mathrm{~min}$, relative to the total number of dispersing females (individual dispersal index). Both selection treatments (high/low) were compared to their control line within replicates $(1-4) ;{ }^{*} P<0.05$. Numbers under the treatments depict the total number of dispersing females. Total number of examined females (including non-dispersers) was 18 per line

\section{Discussion}

In the original population, timing of dispersal varied greatly between females and dispersal away from a colony took place over a time period of at least $12 \mathrm{~h}$ and often over more than $24 \mathrm{~h}$ (see the Appendix (Electronic supplementary material)). Under these circumstances, seven rounds of artificial selection were exerted on the propensity to leave the colony and the response to selection was determined using two indices. Neither index revealed a consistent response to selection. Overall, the selection treatments do not seem to have affected the dispersal behaviour in the selection lines. Also, no consistent and almost no significant differences in life-history traits or sex ratio were found between the selection lines and control lines. Thus, there is no evidence for correlated responses, which would be expected since we also found no response to selection.

How can we explain the lack of response to selection? Most obviously, the result can point towards a lack or a low amount of additive genetic variation for propensity to leave the colony. If this is the case, then the decision to leave the colony is predominantly condition-dependent and does not differ genetically between individuals. However, four aspects of the experimental set-up may have played a confounding role. First, differences in age of the females may have obscured genetic differences. Females that were born earliest in the age cohort (of $24 \mathrm{~h}$ ) may be the first ones to disperse. Almost all females in a colony had reached adulthood before dispersal was observed (pers. obs. Tien), but perhaps newly emerged females must first feed and build a reserve before commencing dispersal. If so, then we may have also selected on age, which would have caused a weaker response to selection. Second, social behaviour may have obscured genetic differences, since females follow the silken threads of other females during dispersal (Yano 2008). However, it is only the speed at which females find the dispersal route that increases if silk-marked routes are present; the initiation of dispersal seems independent of the presence of silk routes 


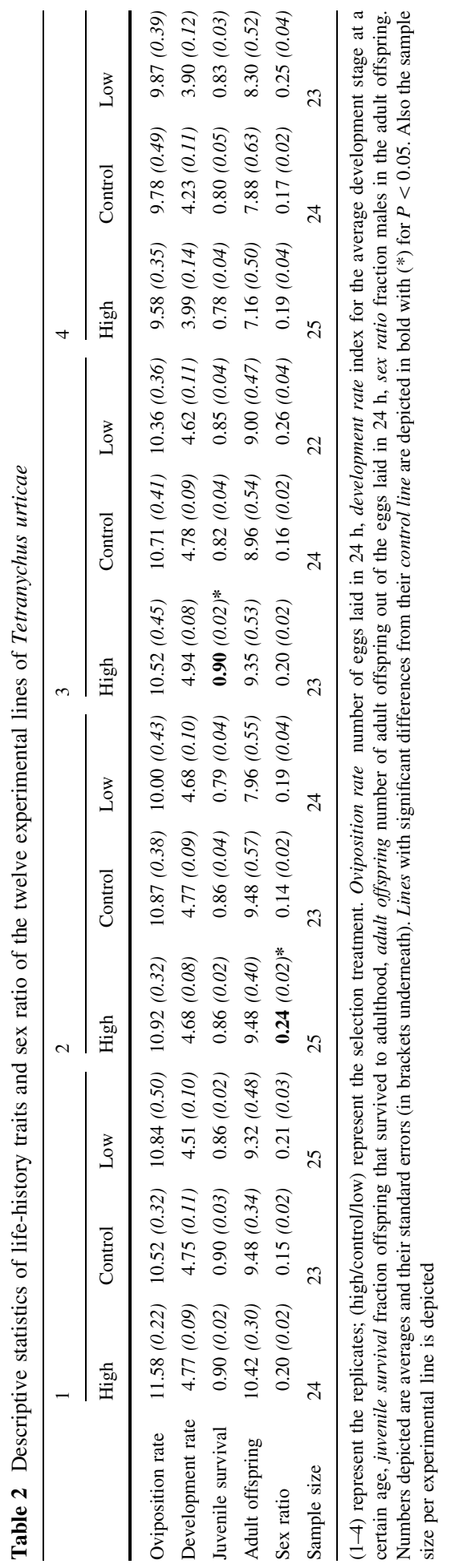


(Fig. 6 in Yano 2008). Third, the downward selection treatment did not entail strong selective forces: on average the females between the 44th and 70th percentile of fastest dispersers were chosen. Thus, selection in the downward treatment was weak which may explain the lack of response in the low lines. However, the upward treatment did involve strong selection and also did not lead to consistent responses. Fourth, the indices used may not be related to the behaviour that was selected on. Selection took place on large groups of individuals (on average 373 females) and using non-standardized conditions of habitat exploitation. Therefore, we used alternative methods to compare the behaviour of the resulting selection lines. One index was based on behaviour of single females, but the behaviour of single females may not be related to behaviour in a colony. However, the experimental set-up for the group index did concern a colony, albeit a small one, and offered a method for dispersal that was similar to the methods provided during the selection experiment (i.e. a bridge to a new leaf). Therefore we assume that the behaviour measured for the group index is related to the behaviour selected on and consequently that the index is appropriate. In conclusion, although we cannot exclude methodological aspects of the experimental set-up partly accounting for a lack of response to selection, it does seem likely that the amount of heritable variation for propensity to disperse is not large. Considering the high selection differentials in especially the high lines for seven generations, if high genetic variation was present some response to selection would be expected despite confounding factors.

Yano and Takafuji (2002) did find a selection response for propensity to leave the colony, using a Japanese population of $T$. urticae. The populations of spider mites used may have had different levels of additive genetic variation, which may explain part of the differences in response. However, elements of the experimental set-up used by Yano and Takafuji probably also contributed to their observed experimental results. Specifically, as explained in the Introduction, a combination of an inappropriate selection regime for the downward selection treatment, inbreeding and/or drift may provide alternative explanations for their reported selection responses. Heritable variation has been found for another aspect of dispersal behaviour in T. urticae, namely the initiation of aerial dispersal (i.e. lifting the anterior part of the body under optimal 'take-off' conditions; (Li and Margolies 1993, 1994). This behaviour in nature takes place from the tops of plants when air currents are present. Given that there is genetic variation for the propensity for aerial dispersal, one could also expect heritable variation for the propensity for ambulatory dispersal. However, we did not find any indication for the latter. Although our experimental set-up improved upon the one by Yano and Takafuji (2002), there are potential weaknesses in our set-up (see above) which may be addressed in future experiments. Further work on dispersal in T. urticae is essential, since dispersal is related to many fundamental aspects of the ecology and evolution of $T$. urticae and other species living in ephemeral habitats, such as the persistence of metapopulations and the evolution of life history traits (e.g., Clobert et al. 2001; Ronce 2007).

Open Access This article is distributed under the terms of the Creative Commons Attribution Noncommercial License which permits any noncommercial use, distribution, and reproduction in any medium, provided the original author(s) and source are credited.

\section{References}

Bolland HR, Gutierrez J, Flechtmann CHW (1998) World catalogue of the spider mite family (Acari: Tetranychidae). Koninklijke Brill NV, Leiden

Brandenburg RL, Kennedy GG (1982) Intercrop relationships and spider mite dispersal in a corn/peanut agro-ecosystem. Entomologia Experimentalis et Applicata 32:269-276 
Clobert J, Danchin E, Dhondt AA, Nichols JD (2001) Dispersal. Oxford University Press, Oxford Crawley MJ (2007) The R Book. Wiley, Chichester

Ellner S, McCauley E, Kendall BE, Briggs CJ, Hosseini PR, Wood SN, Janssen A, Sabelis MW, Turchin P, Nisbet RM, Murdocht WW (2001) Habitat structure and population persistence in an experimental community. Nature 412:538-543

Fjerdingstad EJ, Schtickzelle N, Manhes P, Gutierrez A, Clobert J (2007) Evolution of dispersal and life history strategies-Tetrahymena ciliates. BMC Evol Biol 7:133

Fry JD (1989) Evolutionary adaptation to host plants in a laboratory population of the phytophagous mite Tetranychus urticae Koch. Oecologia 81:559-565

Helle W, Sabelis MW (1985) Spider Mites. Their biology, natural enemies and control. Elsevier, Amsterdam

Hussey NW, Parr WJ (1963) Dispersal of the glasshouse red spider mite Tetranychus urticae Koch (Acarina, Tetranychidae). Entomol Exp Appl 6:207-214

Jeppson LR, Keifer HH, Baker EW (1975) Mites injurious to economic plants. University California Press, Berkeley

Kennedy GG, Smitley DR (1985) Dispersal. In: Helle W, Sabelis MW (eds) World crop pests: spider mites. Their biology, natural enemies and control. Elsevier, Amsterdam, pp 233-242

Li J, Margolies DC (1993) Quantitative genetics of aerial dispersal behaviour and life-history traits in Tetranychus urticae. Heredity 70:544-552

Li J, Margolies DC (1994) Responses to direct and indirect selection on aerial dispersal behaviour in Tetranychus urticae. Heredity 72:10-22

Margolies DC, Kennedy GG (1985) Movement of the two-spotted spider mite, Tetranychus urticae, among hosts in a corn-peanut agroecosystem. Entomologia Experimentalis et Applicata 37:55-61

Mitchell R (1970) An analysis of dispersal in mites. Am Nat 104:425-431

Olivieri I, Couvet D, Gouyon PH (1990) The genetics of transient populations-research at the metapopulation level. Trends Ecol Evol 5:207-210

R-Development-Core-Team (2009) R: A language and environment for statistical computing in R-Foundation-for-Statistical-Computing, ed, Vienna, Austria

Roff DA (2002) Life history evolution. Sinauer Associates Inc., Sunderland

Roff DA, Fairbairn DJ (2001) The genetic basis of dispersal and migration, and its consequences for the evolution of correlated traits. In: Clobert J, Danchin E, Dhondt AA, Nichols JD (eds) Dispersal. Oxford University Press, Oxford, pp 191-202

Ronce O (2007) How does it feel to be like a rolling stone? Ten questions about dispersal evolution. Annu Rev Ecol Evol Syst 38:231-253

Ronce O, Olivieri I (1997) Evolution of reproductive effort in a metapopulation with local extinctions and ecological succession. Am Nat 150:220-249

Sabelis MW (1991) Life-history evolution of spider mites. In: Schuster R, Murphy PW (eds) The Acari: reproduction, development and life-history strategies. Chapman and Hall, London, pp 23-49

Smitley DR, Kennedy GG (1985) Photo-oriented aerial-dispersal behaviour of Tetranychus urticae (Acari, Tetranychidae) enhances escape from the leaf surface. Ann Entomol Soc Am 78:609-614

Sokal RR, Rohlf FJ (1995) Biometry. The principles and practice of statistics in biological research. Freeman and Company, New York

Tien NSH, Sabelis MW, Egas M (2009) The heritability of defense and life-history traits in the two-spotted spider mite. Evol Ecol Res 11:1271-1281

Yano S (2008) Collective and solitary behaviors of twospotted spider mite (Acari : tetranychidae) are induced by trail following. Ann Entomol Soc Am 101:247-252

Yano S, Takafuji A (2002) Variation in the life-history pattern of Tetranychus urticae (Acari: Tetranychidae) after selection for dispersal. Exp Appl Acarol 27:1-10

Yano S, Wakabayashi M, Takabayashi J, Takafuji A (1998) Factors determining the host plant range of the phytophagous mite, Tetranychus urticae (Acari : Tetranychidae): a method for quantifying host plant acceptance. Exp Appl Acarol 22:595-601 